\title{
Efficacy and Safety of Metformin for Treatment of Overweight and Obesity in Adolescents: An Updated Systematic Review and Meta-Analysis
}

\author{
Carmen Bouza Teresa López-Cuadrado Luisa Fernanda Gutierrez-Torres \\ JoséMaria Amate \\ Health-Care Technology Assessment Agency, Instituto de Salud Carlos III, Madrid, Spain
}

Key Words

Efficacy $\cdot$ Safety $\cdot$ Metformin $\cdot$ Obesity $\cdot$ Adolescents $\cdot$ Meta-Analysis

\begin{abstract}
Objective: To assess the efficacy and safety of using metformin in overweight and obese adolescents without related morbidity. Methods: We conducted a systematic review and meta-analysis of randomized clinical trials published until June 2011, comparing metformin to placebo or other interventions. Our primary variables were baseline BMI changes and development of adverse effects. Results: Nine studies (498 participants, mean age 14.2 years; mean BMI $36.4 \mathrm{~kg} / \mathrm{m}^{2}$ ) met the inclusion criteria. In all but one study, metformin was combined with lifestyle modification and was compared to placebo combined with changes in lifestyle. Analysis showed that metformin reduced mean BMI by $1.42 \mathrm{~kg} / \mathrm{m}^{2}(95 \% \mathrm{CI}-2.18,0.66)$, fasting insulin by $9.9 \mu \mathrm{U} / \mathrm{ml}(95 \% \mathrm{CI}-13.8,-6.06)$ and the HOMA index by $-1.78(95 \% \mathrm{CI}-3.32,-0.23)$. No changes in any other parameter were observed. No statistical differences were found in the rate of adverse events (33\% metformin, $32 \%$ placebo).The withdrawal rates due to side effects in the metformin and placebo groups were $2.7 \%$ and $2.5 \%$, respectively. Conclusions: The available evidence indicates that, in the short term, administration of metformin in addition to lifestyle modification is relatively effective for reducing BMI and hyperinsulinemia among obese adolescents without related morbidity, and displays an acceptable safety pattern. Nevertheless, its long-term impact is unknown.


Bouza et al.: Efficacy and Safety of Metformin for Treatment of Overweight and

Obesity in Adolescents: An Updated Systematic Review and Meta-Analysis

\section{Introduction}

The enormous individual and social burden of adolescent overweight and obesity makes it one of today's greatest public health problems and a challenge to all heathcare systems [1-2]. Defined as BMI equal to or greater than the 85th or 95th percentiles and adjusted for age and sex [3], prevalence has doubled or even tripled since the 1970s in most industrialized and many developing countries [1-3].

Apart from its impact, both psychosocially and on quality of life [4], obesity in adolescence is associated with metabolic complications [5] and leads to a substantially increased risk of chronic comorbidities and premature death [6,7]. Of overweight and obese adolescents, $50-72 \%$ present with reduced insulin sensitivity [5], which determines an increase in the prevalence of metabolic syndrome and type 2 diabetes not only in minority groups but also in the general population [7].

The current standard treatment is lifestyle modification and essentially seeks to attain the twin goals of balanced diet and physical exercise $[8,9]$. Yet, most of the therapeutic approaches fail to achieve any major, sustained weight loss, even in the best multidisciplinary programs [10-12]. This is due, in part, to difficulties in adherence and the multiplicity of biological and social factors which intervene in the development of obesity $[10,13]$. The insufficient effect of current therapeutic approaches also encourages the search for adjuvant drug treatments.

Metformin has been shown to be relatively effective in reducing weight and improving insulin sensitivity in adults and is one of the drugs proposed for treatment of overweight and obesity among children and adolescents who do not respond to changes in lifestyle or present with insulin resistance $[10,12,14]$. However, there are doubts about prescribing a drug whose efficacy and safety in this population have not been clearly demonstrated [15].

Nevertheless, the use of metformin among overweight and obese non-diabetic children and adolescents is steadily increasing in clinical practice $[12,15]$ despite the fact that this drug has not been authorized for this indication and that the literature on its efficacy and safety is inconsistent [11,16-18]. Thus, the results of a number of systematic reviews ranged from reporting the absence of any significant efficacy [16] to reporting modest favorable effects on BMI [17] that are limited to some adolescents with clinical resistance to insulin or pre-diabetes [18]. Most of them agreed that more data are necessary to determine the role of metformin in the management of pediatric obesity $[11,18,19]$.

In such a scenario, we sought to update the evidence on the efficacy and safety of metformin for treating obesity in adolescents by conducting a systematic review and metaanalysis of the literature that attempted to answer the following questions:

i) What are the results of metformin treatment in overweight and obese adolescents as compared to placebo or other forms of treatment?

ii) Do these effects persist when the treatment is halted?

iii) Does the treatment have effects other than weight loss?

iv) What are the adverse effects of metformin as compared to placebo or other forms of treatment?

\section{Methods}

A systematic review of the literature published until June 2011 was conducted in the following electronic databases: OVID Medline Embase; ISI Web of Knowledge and The Cochrane Library. In every case MesH terms ('Metformin' AND 'Obesity' or 'Overweight'), were used with specific filters to select participants' age range ( $\geq 12$ to $\leq 19$ years) [9] and 
study design (randomized controlled trial) [20], without any language restrictions. Similarly, a manual search was made of relevant references [20], and experts were contacted to identify unpublished studies. In no case contact was made with the industry.

\section{Inclusion and Exclusion Criteria}

We included any randomized clinical trial that met the following criteria: i) population: trials conducted on adolescents with formal diagnosis of overweight or obesity; ii) intervention: oral administration of metformin; iii) comparator: placebo or any other treatment; iv) results: inclusion of objective data of at least one of our primary variables of efficacy and safety, namely, change in baseline BMI and development of adverse effects. Metabolic and biochemical parameters, including insulin resistance markers, were deemed to be secondary variables.

The selection of BMI $\left(\mathrm{kg} / \mathrm{m}^{2}\right)$ as the primary variable was based on its status as the preferred clinical measure of overweight in adolescents $[3,9,21]$ and its correlation with the development of complications $[8,10]$. Likewise, it is widely recommended for measuring the efficacy of specific interventions on obesity $[3,9,11]$.

Studies in which the participants presented with comorbidities (e.g., type 1 or type 2 diabetes, polycystic ovary syndrome, metabolic syndrome, neoplasms, steroid treatment, etc.) were excluded, as were nonrandomized and quasi-randomized studies. In the case of crossover trials, these were also excluded unless the paper furnished data on the stage prior to crossover, in which case only such data were used.

Furthermore, no limitations were placed on study duration, which was defined as shortterm (up to 12 months after inclusion) or medium-term (1-5 years after inclusion in the study or at least 12 months since termination of treatment).

\section{Study Selection}

The studies located were examined by two independent reviewers. Points of disagreement were settled by discussion of the data from the study in question.

\section{Data Extraction}

Original data were extracted on a standard form that included: i) details of the study design, exclusion/inclusion criteria, and duration of follow-up; ii) information about the study population; iii) information about the intervention and comparator; iv) information about the outcome measures of efficacy and safety.

The variable BMI, was extracted as described in each original study, with this measure taking different forms, i.e., final BMI, absolute change in BMI over baseline, percentage change over baseline value, absolute change in the standard deviations of BMI (SDS BMI) or baseline z-scores. Where any study furnished more than one outcome measure, this was selected in the following order: BMI as the measure most frequently described in adolescents [11], BMI percentile, and BMI z-score [9].

\section{Analysis of Methodological Quality and Scientific Evidence}

This was performed in accordance with validated recommendations [20].

\section{Data Analysis and Summary of Results}

A critical assessment was made of each study's methodological quality and results. The existence of clinical heterogeneity was examined via a thorough analysis of the inclusion and exclusion criteria as well as the baseline characteristics of the respective study populations. Because studies varied considerably in characteristics that can affect both baseline and post-intervention BMI $[9,20]$, the inverse variance method with a random effects model 


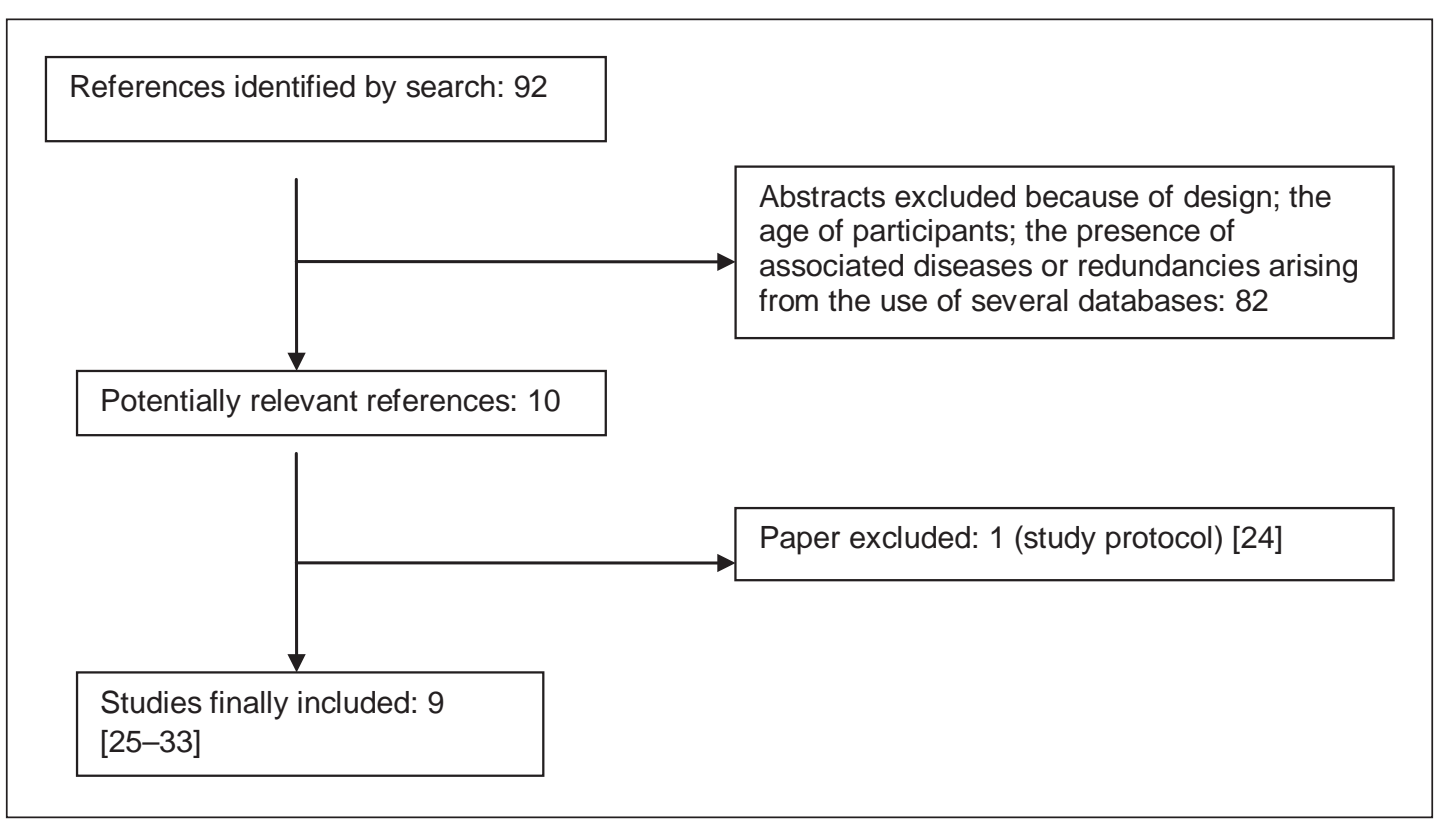

Fig. 1. Flow diagram for study selection and inclusion.

was used in the meta-analysis. Dichotomous variables were analyzed by estimation of relative risk (RR) with its $95 \%$ confidence interval $(95 \% \mathrm{CI}$ ), and continuous variables by difference in means with its $95 \% \mathrm{CI}$. The degree of inconsistency among studies was assessed using $\mathrm{I}^{2}$ statistics, with a value $>50 \%$ taken as relevant [22]. Sensitivity analyses were performed to examine statistical inconsistency, and subgroup analysis was done to compare the studies in which metformin was associated with lifestyle interventions against the only study in which metformin was not associated with such interventions.

Owing to their limitations [23], funnel-plots were not used to examine publication bias.

All analyses were performed using the STATA ${ }^{\circledR}$ IC Stata 11 computer software package (StataCorp LP College Station, TX, USA). Results were deemed significant at a p value $<0.05$.

\section{Results}

The search of the above-mentioned databases identified 92 references, 10 of which fulfilled the inclusion criteria (fig. 1). One of the papers was subsequently excluded because it was a study protocol [24]. Hand searching of retrieved articles yielded no additional studies, and no unpublished randomized trials were identified. Finally, 9 randomized clinical trials [25-33] met the inclusion criteria (table 1).

The studies selected included 498 participants, 295 of whom comprised the metformin group and 203 the control or placebo group. Participants had a mean BMI of $36.4 \mathrm{~kg} / \mathrm{m}^{2}$ and presented with no related comorbidities or pathological causes of obesity. Most of the studies incorporated insulin sensitivity disorders as an inclusion criterion; the mean age of the adolescents included was 14.2 years. Metformin dosage ranged from 1,000 to 2,000 $\mathrm{mg} / \mathrm{day}$, and all but one study [26] included changes in participants' lifestyles, though the intensity of such changes varied. Hence, in some studies, lifestyle modification consisted of specific and individualized, structured programs with involvement of the family group, 


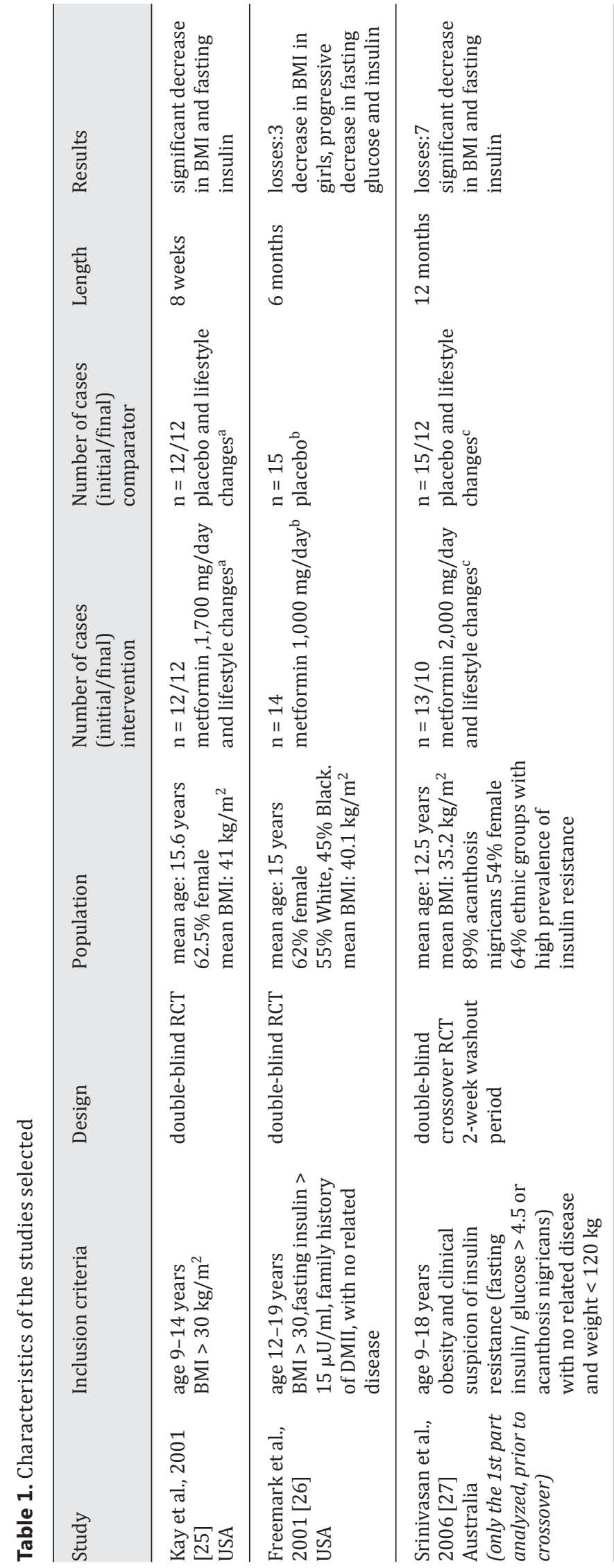

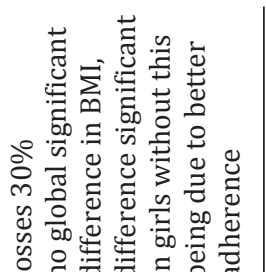

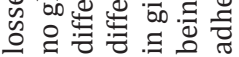

Ẽ
है
हू

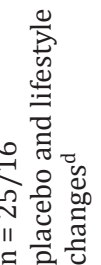

त्

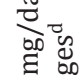

은

雍空

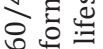

II

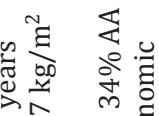



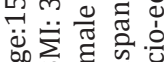

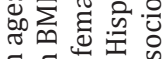

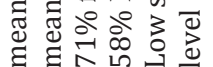

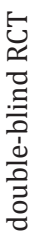

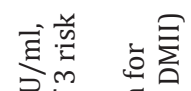

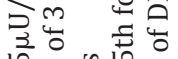
내는

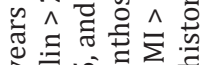

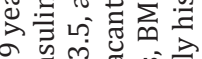

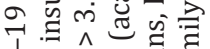

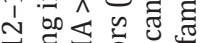

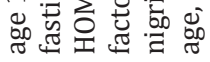

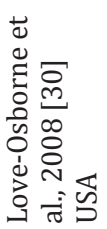

www.karger.com/ofa

Treatment of Overweight and

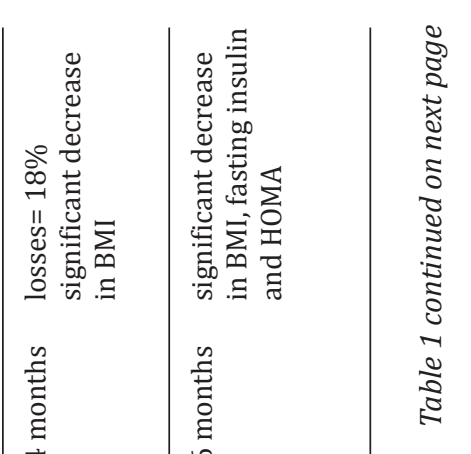




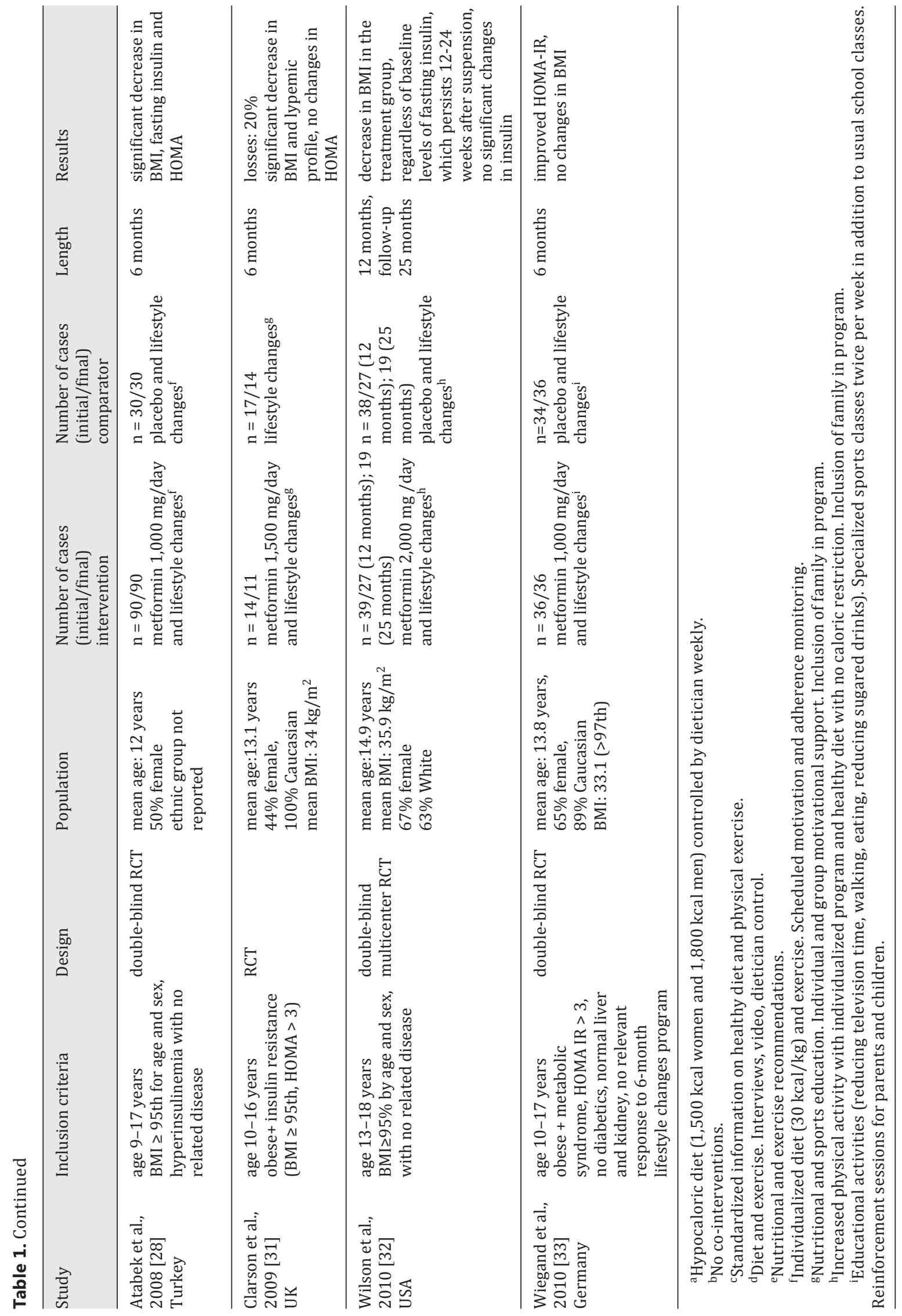


Table 2. Methodological quality of included studies

\begin{tabular}{|c|c|c|c|c|c|c|c|}
\hline Study & $\begin{array}{l}\text { Concealment in } \\
\text { allocation to } \\
\text { treatment }\end{array}$ & $\begin{array}{l}\text { Adequate } \\
\text { randomisation }\end{array}$ & Blinding & $\begin{array}{l}\text { Reasons for } \\
\text { withdrawal }\end{array}$ & $\begin{array}{l}\text { Incomplete } \\
\text { data }\end{array}$ & $\begin{array}{l}\text { Free of } \\
\text { other biases }\end{array}$ & Risk of bias \\
\hline Kay et al. [25] & NR & NR & yes & NR & no & yes & moderate \\
\hline Freemark et al. [26] & NR & NR & yes & reported & yes & yes & moderate \\
\hline Srinivasan et al. [27] & adequate & yes & yes & reported & no & yes & low \\
\hline Love-Osborne et al. [30] & adequate & yes & yes & NR & yes & yes & moderate \\
\hline Burgert et al. [29] & adequate & yes & yes & reported & no & yes & low \\
\hline Atabek et al. [28] & adequate & not clear & yes & NR & yes & doubtful & moderate \\
\hline Clarson et al. [31] & adequate & yes & doubtful & reported & no & yes & moderate \\
\hline Wilson et al. [32] & adequate & yes & yes & reported & no & yes & low \\
\hline Wiegand et al. [33] & adequate & yes & yes & reported & no & yes & low \\
\hline
\end{tabular}

NR $=$ Not reported .

regular reinforcement, and follow-up [28, 30,33], whereas in others the intervention was less intense and less rigorous [25, 27, 29]. Duration of treatment ranged from 2 [25] to 12 months [32] and was 6 months in most cases. The principal characteristics of the studies selected are shown in table 1.

Regarding the methodological quality, risk of bias can be categorized as low or moderate (table 2). In 7 studies, concealment in allocation to treatment appeared to be adequate, as was randomization. Only 1 study [31] made no clear reference to blinding, and three failed to describe the reasons for withdrawal from the study [27, 28, 30]. Nevertheless, only 1 study reported that the analysis of results was performed on an intention-to-treat basis [32].

Of the patients enrolled, 436 (88\%) finished the study, with mean adherence rates of $89 \%(263 / 295)$ in the metformin group and $85 \%$ (173/203) in the control group.

\section{Efficacy}

\section{$B M I$}

Joint analysis of the 8 studies that furnished data on change in BMI values from baseline to termination of treatment showed a significant reduction versus placebo (fig. 2), with an overall decrease of $1.42 \mathrm{~kg} / \mathrm{m}^{2}(95 \% \mathrm{CI}-2.18,-0.66)$. Note should, however, be taken of the presence of a notable degree of inconsistency among these studies, which is reflected in an $I^{2}$ value of $65 \%$.

A sensitivity analysis that excluded the study whose baseline BMI corresponded to an overweight status [28] led to a notable reduction in study inconsistency $\left(I^{2} 47 \%, p=0.08\right)$, with the global estimator remaining significantly favorable to metformin, albeit with a slight decrease in magnitude (WMD: -1.15 (95\% CI -1.84, -0.48). In order to identify other possible causes of the statistical heterogeneity observed, sensitivity analyses were additionally performed by reference to studies' methodological quality and risk of bias (low vs. moderate), metformin dose ( $<1,700 \mathrm{vs.} \geq 1,700 \mathrm{mg} /$ day) and duration of treatment ( $<6 \mathrm{vs}$. $\geq 6$ months), without the degree of inconsistency being reduced in any case. Similarly, an attempt was also made to perform an analysis according to the ethnic and cultural characteristics of the population included, which proved impossible due to the absence of specific data in individual studies. The only analysis that could be performed was by continent of 


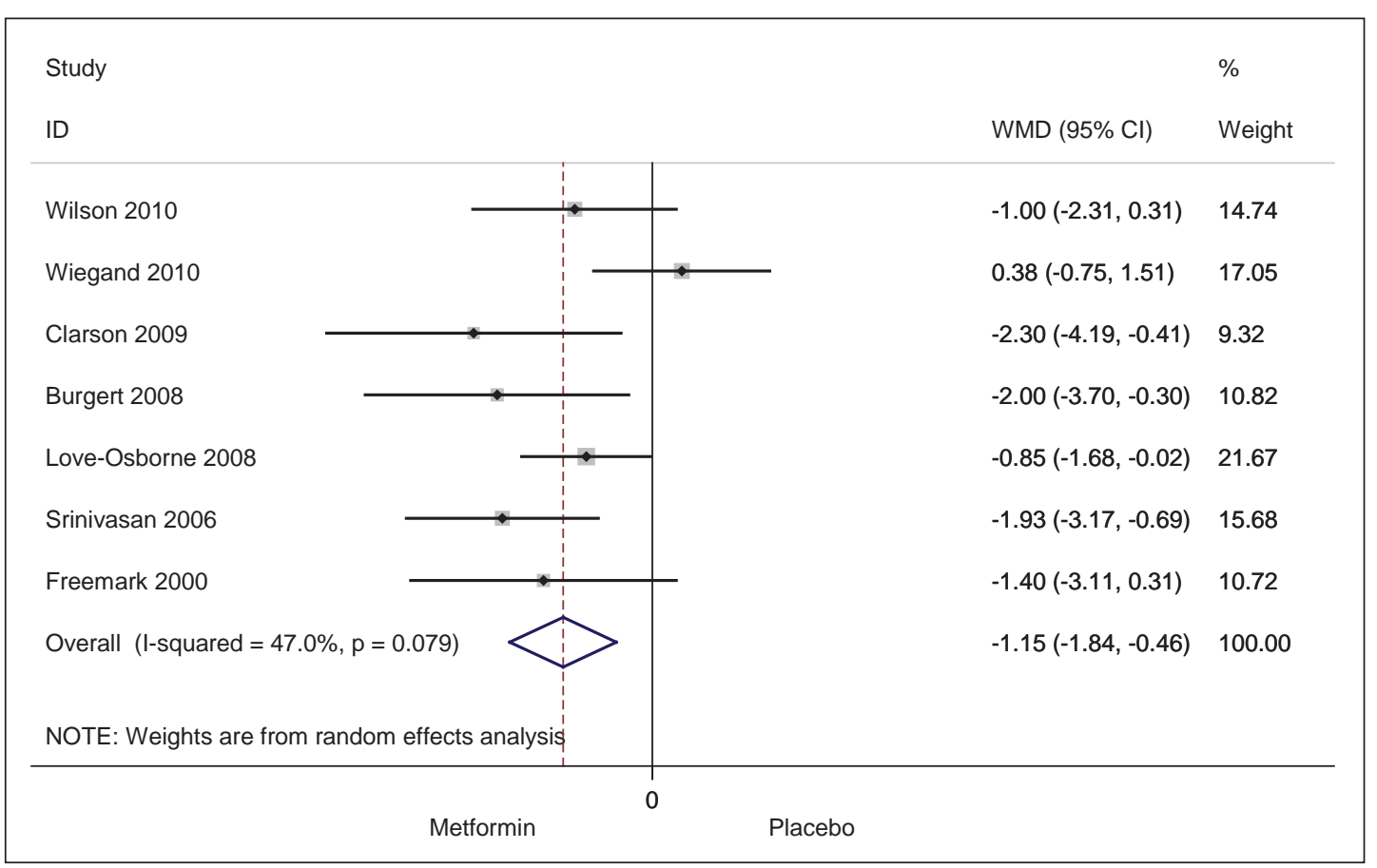

Fig. 2. Changes in BMI values: results of meta-analysis (all based on random effects meta-analysis).

study implementation, the results of which indicated a high degree of consistency $\left(\mathrm{I}^{2}=0 \%\right)$ among the 5 studies conducted in North America [26, 29-32] and an estimator of -1.21 (95\% CI $-1.79,-0.63)$.

The efficacy of metformin was also verified by analysis of BMI z-scores, with an overall value of -0.09 ( $95 \%$ CI $-0.14,-0.04)$. Although there were only 4 studies that furnished data $[27,31-33]$, no heterogeneity was observed among them $\left(\mathrm{I}^{2}=0\right)$.

\section{Metabolic Parameters}

Only 4 studies [25, 28, 29, 31] afforded data on fasting glucose and, when analyzed, showed that metformin failed to significantly modify fasting glucose levels. Furthermore, joint analysis of the 6 studies providing data [25-30], indicated that metformin had a favorable effect on fasting insulin levels, with a reduction of $9.94 \mu \mathrm{U} / \mathrm{ml}$ (95\% CI-13.8 -6.06) and no significant heterogeneity among the individual studies $\left(\mathrm{I}^{2}=42 \%, \mathrm{p}=0.125\right)$. As regards the HOMA index, though analysis of the 7 studies with this data [25-32] showed that metformin significantly reduced this parameter (overall effect -1.78 (95\% CI -3.32 , $-0.23)$, there was a considerable degree of heterogeneity among these studies $\left(\mathrm{I}^{2}=90.3 \%\right.$, $\mathrm{p}=0.03$ ). Unfortunately, despite the different sensitivity analyses performed, the cause $(\mathrm{s})$ of this heterogeneity could not be identified.

With respect to cholesterol-related effects, after analysis of the 5 studies with data on this variable $[25,26,28,29,33]$ no significant differences were observed between metformin and placebo in terms of total cholesterol levels (weighted mean difference -2.78 (95\% CI -8.41, 2.85; $\mathrm{I}^{2}=25.3 \%$ ) or high-density lipoprotein (analysis of 5 studies, overall estimator 0.21 $(95 \%$ CI $-3.07,2.66)$ and low-density lipoprotein fractions (combined analysis of 6 studies, effect estimator $0.55\left(95 \% \mathrm{CI}-3.32,4.41 ; \mathrm{I}^{2}=0 \%\right)$. Similarly, there was no significant effect in terms of change in triglyceride levels (overall effect -29.21 (95\% CI -60.47,2.05), a finding that was accompanied by an important degree of inconsistency among the studies $\left(\mathrm{I}^{2}=86 \%\right)$. 


\begin{tabular}{|c|c|c|c|}
\hline Study & & & $\%$ \\
\hline ID & & $\operatorname{RR}(95 \% \mathrm{Cl})$ & Weigh \\
\hline Atabek 2008 & & $1.70(0.08,34.52)$ & 6.02 \\
\hline Burgert 2008 & & $1.00(0.02,47.55)$ & 3.90 \\
\hline Freemark 2001 & & $11.73(0.71,194.51)$ & 6.75 \\
\hline Kay 2001 & & $15.00(0.95,236.42)$ & 6.96 \\
\hline Love-Osborne 2008 & & $2.06(0.65,6.55)$ & 21.05 \\
\hline Srinivasan 2006 & & $5.71(0.30,109.22)$ & 6.22 \\
\hline Wiegand 2010 & & $0.52(0.20,1.41)$ & 23.88 \\
\hline Wilson 2010 & & $2.81(1.13,7.00)$ & 25.22 \\
\hline Overall $(I-$ squared $=39.7 \%, p=0.114)$ & & $2.12(0.95,4.76)$ & 100.00 \\
\hline \multicolumn{4}{|l|}{ NOTE: Weights are from random effects analysis } \\
\hline & & & \\
\hline Placebo & Metformin & & \\
\hline
\end{tabular}

Fig. 3. Gastrointestinal adverse events: results of meta-analysis (all based on random effects meta-analysis).

\section{Safety}

In the 8 studies [25-30,32,33] that furnished data, development of adverse effects was described in 33\% (94/280) of subjects treated with metformin and in 32\% (60/187) of those treated with placebo, without the difference attaining statistical significance (RR 1.05, 95\% CI $0.8,1.36, I^{2}=48.3 \%$ ). In much the same way, patients treated with metformin registered a higher risk of adverse gastrointestinal effects than the control group, but the difference once again failed to reach statistical significance (fig. 3).

The withdrawal rate in the metformin $(30 / 292,10.3 \%)$ versus the placebo group $(37 / 203,15 \%)$ was significantly favorable to metformin (RR $0.65,95 \%$ CI $0.42,0.99, I^{2}=0 \%$ ). Losses were linked to social factors, such as change of address or school, unavailability of parents for study compliance purposes, or presence of adverse effects. In respect of this last mentioned factor, there were no significant differences between the metformin $(8 / 292$; $2.7 \%$ ) and placebo groups (5/203; 2.5\%) (RR 1.04, 95\% CI 0.39, 2.78, $\mathrm{I}^{2}=0 \%$ ).

\section{Discussion}

This systematic review - to our knowledge the first of its kind targeting on adolescents exclusively - identified 9 randomized clinical trials of adequate methodological quality, which addressed the efficacy and safety of metformin combined with lifestyle interventions in the management of adolescent obesity. Their results indicate that among obese adolescents with no associated diseases, administration of metformin is relatively effective in the 
short term for reducing BMI and variables of insulin resistance (fasting insulin, HOMA), and likewise displays an acceptable safety pattern.

Control of obesity and its potential complications in adolescents poses a challenge to all healthcare systems. In the absence of intervention, overweight and obese adolescents display a continuous trend towards more rapid weight gain than normal, with BMI being acknowledged as the major risk factor for development of complications and, thus, the most important variable for measuring the efficacy of specific interventions $[6,7,9]$. This metaanalysis shows that in the short term treatment with metformin, in tandem with individualized, structured lifestyle modification programs, achieves a reduction in BMI that, albeit modest, is significantly greater than that achieved by lifestyle modification alone. The results of a worthy, recent systematic review indicate that, at 12 months of intervention, medium to high intensity lifestyle modification programs achieve a 1.9 to $3.3 \mathrm{~kg} / \mathrm{m}^{2}$ reduction in BMI versus the comparator [9]. In our study, the addition of metformin to changes in lifestyle led to a reduction in BMI of around 1.21 to $1.42 \mathrm{~kg} / \mathrm{m}^{2}$. It must be said that, while this effect might be modest in size, it was nevertheless achieved among adolescents who had often shown themselves to be refractory to lifestyle modification, something that favors the use of metformin in this population group. These data, which extend and enlarge those obtained by Park et al. [17] after analyzing 5 studies with 320 participants, are in line with those reported by other authors who not only highlight the fact that, in many cases and even in the best multiprofessional program scenarios, changes in lifestyle are not enough among adolescents because they fail to achieve effective, sustained weight loss, but also single out the adjuvant role of pharmacological agents [10-12].

It should however be noted that the follow-up time of these studies was relatively short and that there are no medium- to long-term data on metformin's efficacy on BMI.

With respect to the second question regarding the persistence of the drug's effects after treatment has ended, it would seem that, despite the paucity of data, the effects of metformin tend to disappear post treatment. Indeed, only 1 study [32] analyzed changes in BMI 1 year after termination of treatment. In this study, the differences between the groups were observed to persist in the first 12-24 weeks after suspension of the drug. Thereafter, the effect disappeared, and the mean BMI of the group treated with metformin came to resemble that of the control group, with no significant differences being observable between the two groups.

Insofar as insulin sensitivity markers are concerned, our results show that, whereas metformin significantly improves fasting insulin levels, no significant changes are observed in glucose levels. Although the reduction in insulin levels is in accordance with the literature on the drug [34], it could also be linked to the significant weight loss documented among patients in the treatment group [35]. The result seems relevant to us because, despite differences in the populations included and metformin dosages used and despite changes and variability in insulin sensitivity during puberty [36], the studies display a notable degree of consistency. In the context of the above data, the combined analysis of the HOMA index is significant for metformin. Nevertheless, the individual studies clearly displayed a wide degree of heterogeneity which remained in evidence after the various sensitivity analyses had been conducted; thus caution is called for when it comes to interpreting this finding. While one cannot say for certain what the precise cause of such heterogeneity might be, it could perhaps lay in intra and interlaboratory variability in calculation of the insulin resistance indices, since there is no reliable standard method for their determination at present [18].

The remaining parameters studied, i.e., lipid and triglyceride profiles, showed no statistically significant changes. In both cases, one cannot rule out that this result may be due to the fact that the values are within the normal range, since the effects of metformin on plasma cholesterol and triglycerides are inconsistent and appear to depend on the presence of dyslipidemia [37]. 
In terms of drug safety, our meta-analysis showed that, from a statistical standpoint, there were no significant differences in the frequency of adverse effects between the metformin and placebo groups. Similarly, no differences were observed between the groups in terms of withdrawals from the study due to side effects.

The principal side effects associated with metformin were gastrointestinal problems, though no statistically significant differences between the metformin and control groups in the presentation of these disorders were observed. These data confirm those previously described in the literature on metformin.

Lastly, the higher rate of adherence seen in the group treated with metformin opens up the possibility that the drug may improve adherence to lifestyle modification programs.

\section{Limitations}

As with all systematic reviews, there is the possibility that our study may not have identified some relevant study whose potential impact on the results cannot be estimated. To reduce this risk, however, we conducted a wide-ranging bibliographic search that included various electronic databases, made an intense manual search of references, and contacted experts on the subject so that we feel to have minimized any possible risk. Moreover, as stated in the 'Methods' section, it should be stressed that at no time contact was made with the industry.

Even so, this study is limited by a number of characteristics of the scientific literature, in particular the low number of structured studies published, their small sample size, and short time of follow-up, with these all being short-term studies having a maximum length of 1 year and thus being unable to reflect the natural history of the process. In addition, it is essential to underscore the lack of uniformity in the methodology used in determining the insulin resistance variables and the units in which these are described. Likewise, the absence of specific results by reference to data of relevance (e.g., sex, ethnic group, and socioeconomic level) in individual studies hindered our conducting an analysis stratified according to such variables, which might in turn have affected treatment outcomes. Furthermore, though their overall methodological quality was observed to be acceptable, with little or no risk of bias, only 1 of the studies conducted an analysis by intention-to-treat.

Lastly, sight should not be lost of the fact that these are clinical trials undertaken on very carefully selected overweight and obese adolescents, which limits extrapolation of their results to other populations.

\section{Conclusions}

Notwithstanding the above mentioned limitations, analysis of the evidence available shows that administration of metformin within the context of a lifestyle modification program seems to be relatively effective and safe for treatment of juvenile obesity in the short term. Nevertheless, nothing is known about the drug's effects, either in the long term or in other, less carefully chosen groups.

Accordingly more robust data are called for, particularly in terms of metformin's medium- to long-term efficacy and safety, which would provide crucial information for clinical and health policy decision making as regards the drug's potential utility for management of the prevalent and vulnerable population of overweight and obese adolescents. 


\section{Acknowledgments}

We would like to thank Zuleika Saz-Parkinson for her technical help with the English version of the manuscript.

Funding for this study was partially provided by a grant from the National R\&D Plan. The funding body had no further role in the study design, in the collection, analysis and interpretation of data, in the writing of the report, and in the decision to submit the paper for publication.

\section{Disclosure Statement}

The authors declare that this study did not receive any support from industry or private corporations, and that there is no potential conflict of interest with respect to metformin or any other therapeutic agent for obesity that could be perceived as prejudicing the impartiality of the research reported. The authors have no affiliations with any organization or entity with a financial interest in or financial conflict with the subject matter or materials discussed in the manuscript.

\section{References}

1 Wang Y, Lobstein T: Worldwide trends in childhood overweight and obesity. Int J Pediatr Obes 2006;1: $11-25$.

2 Lobstein T, Baur L, Uauy R, IASO International Obesity TaskForce: Obesity in children and young people: a crisis in public health. Obes Rev 2004;5(suppl 1):4-85.

3 Himes JH, Dietz WH: Guidelines for overweight in adolescent preventive services: recommendations from an expert committee. The Expert Committee on Clinical Guidelines for Overweight in Adolescent Preventive Services. Am J Clin Nutr1994;59:307-316.

- 4 Wille N, Bullinger M, Holl R, Hoffmeister U, Mann R, Goldapp C, Reinehr T, Westenhöfer J, Egmond-Froehlich A, Ravens-Sieberer U: Health-related quality of life in overweight and obese youths: results of a multicenter study. Health Qual Life Outcomes 2010;8:36.

5 Lee JM, Okumura M J, Davis MM, Herman WH, Gurney JG: Prevalence and determinants of insulin resistance among U.S. adolescents: a population-based study. Diabetes Care 2006;29:2427-2432.

- 6 Bjørge T, Engeland A, Tverdal A, Smith GD: Body mass index in adolescence in relation to cause-specific mortality: a follow-up of 230,000 Norwegian adolescents. Am J Epidemiol 2008;168:30-37.

- 7 Tirosh A, Shai I, Afek A, Dubnov-Raz G, Ayalon N, Gordon B, Derazne E, Tzur D, Shamis A, Vinker S, Rudich A: Adolescent BMI trajectory and risk of diabetes versus coronary disease. N Engl J Med 2011;364:13151325.

8 Spear BA, Barlow SE, Ervin C, Ludwig DS, Saelens BE, Schetzina KE, Taveras EM: Recommendations for treatment of child and adolescent overweight and obesity. Pediatrics 2007;120(suppl 4):S254-288.

- 9 Whitlock EP, O'Connor EA, Williams SB, Beil TL, Lutz KW: Effectiveness of weight management interventions in children: a targeted systematic review for the USPSTF. Pediatrics 2010;125:e396-418.

10 Freemark M: Pharmacotherapy of childhood obesity: an evidence-based, conceptual approach. Diabetes Care 2007;30:395-402.

11 Oude Luttikhuis H, Baur L, Jansen H, Shrewsbury VA, O’Malley C, Stolk RP, Summerbell CD: Interventions for treating obesity in children. Cochrane Database Syst Rev 2009;21:CD001872.

12 Hearnshaw C, Matyka K: Managing childhood obesity: when lifestyle change is not enough. Diabetes Obes Metab 2010;12:947-957.

13 Caprio S, Daniels SR, Drewnowski A, Kaufman FR, Palinkas LA, Rosenbloom AL, Schwimmer JB: Influence of race, ethnicity, and culture on childhood obesity: implications for prevention and treatment: a consensus statement of Shaping America's Health and the Obesity Society. Diabetes Care 2008;31:2211-2221.

14 Caprio S: Treatment of impaired glucose tolerance in childhood. Nat Clin Pract Endocrinol Metab 2008;4: 320-321.

15 Goldfarb B: Pediatric endocrinology debate prescribing drugs to teens with pre-diabetes. DOC News 2007; 4:15-16.

16 McGovern L, Johnson JN, Paulo R, Hettinger A, Singhal V, Kamath C, Erwin PJ, Montori VM: Treatment of pediatric obesity: a systematic review and meta-analysis of randomized trials. J Clin Endocrinol Metab 2008;93:4600-4605.

17 Park MH, Kinra S, Ward KJ, White B, Viner RM: Metformin for obesity in children and adolescents: a systematic review. Diabetes Care 2009;32:1743-1745. 
Quinn SM, Baur LA, Garnett SP, Cowell CT: Treatment of clinical insulin resistance in children: a systematic review. Obes Rev 2010;11:722-730.

19 Han JC, Lawlor DA, Kimm SY: Childhood obesity. Lancet 2010;375:1737-1748.

20 Higgins JPT, Green S (eds): Cochrane Handbook for Systematic Reviews of Interventions Version 5.0.2 (updated September 2009). www.cochrane-handbook.org.

-21 Dietz WH, Robinson TN: Use of the body mass index (BMI) as a measure of overweight in children and adolescents. J Pediatr 1998;132:191-193.

22 Higgins JPT, Thompson SG, Deeks JJ, Altman DG: Measuring inconsistency in meta-analyses. BMJ 2003;327: 557-560.

23 Tang J, Liu JL: Misleading funnel plot for detection of bias in meta-analysis. J Clin Epidemiol 2000;53:477484.

24 Wilson AJ, Prapavessis H, Jung ME, Cramp AG, Vascotto J, Lenhardt L, Shoemaker JK, Watson M, Robinson T, Clarson CL: Lifestyle modification and metformin as long-term treatment options for obese adolescents: study protocol. BMC Public Health 2009;30;9:434.

25 Kay JP, Alemzadeh R, Langley G, D’Angelo L, Smith P, Holshouser S: Beneficial effects of metformin in normoglycemic morbidly obese adolescents. Metabolism 2001;50:1457-1461.

-26 Freemark M, Bursey D: The effects of metformin on body mass index and glucose tolerance in obese adolescents with fasting hyperinsulinemia and a family history of type 2 diabetes. Pediatrics 2001;107:e55.

-27 Srinivasan S, Ambler GR, Baur LA, Garnett SP, Tepsa M, Yap F, Ward GM, Cowell CT: Randomized, controlled trial of metformin for obesity and insulin resistance in children and adolescents:improvement in body composition and fasting insulin. J Clin Endocrinol Metab 2006;91:2074-2080.

-28 Atabek ME, Pirgon 0: Use of metformin in obese adolescents with hyperinsulinemia:a 6-month, randomized, double-blind, placebo-controlled clinical trial. J Pediatr Endocrinol Metab 2008;21:339-348.

-29 Burgert TS, Duran EJ, Goldberg-Gell R, Dziura J, Yeckel CW, Katz S, Tamborlane WV, Caprio S: Short-term metabolic and cardiovascular effects of metformin in markedly obese adolescents with normal glucose tolerance. Pediatr Diabetes 2008;9:567-576.

-30 Love-Osborne K, Sheeder J, Zeitler P: Addition of metformin to a lifestyle modification program in adolescents with insulin resistance. J Pediatr 2008;152:817-822.

-31 Clarson CL, Mahmud FH, Baker JE, Clark HE, McKay WM, Schauteet VD, Hill DJ: Metformin in combination with structured lifestyle intervention improved body mass index in obese adolescents, but did not improve insulin resistance. Endocrine 2009;36:141-146.

-32 Wilson DM, Abrams SH, Aye T, Lee PD, Lenders C, Lustig RH, Osganian SV, Feldman HA: Glaser Pediatric Research Network Obesity Study Group. Metformin extended release treatment of adolescent obesity: a 48-week randomized, double-blind, placebo-controlled trial with 48-week follow-up. Arch Pediatr Adolesc Med 2010;164:116-123.

-33 Wiegand S, l'Allemand D, Hübel H, Krude H, Bürmann M, Martus P, Grüters A, Holl RW: Metformin and placebo therapy both improve weight management and fasting insulin in obese insulin-resistant adolescents: a prospective, placebo-controlled, randomized study. Eur J Endocrinol.2010;163:585-592.

34 Yki-Järvinen H: Thiazolidinediones. N Engl J Med 2004;351:1106-1118.

-35 Reinehr T, Kiess W, Kapellen T, Andler W: Insulin sensitivity among obese children and adolescents, according to degree of weight loss. Pediatrics 2004;114:1569-1573.

-36 Travers SH, Jeffers BW, Bloch CA, Hill JO, Eckel RH: Gender and Tanner stage differences in body composition and insulin sensitivity in early pubertal children. J Clin Endocrinol Metab 1995;80:172-178.

-37 Fontbonne A, Charles MA, Juhan-Vague I, Bard JM, André P, Isnard F, Cohen JM, Grandmottet P, Vague P, Safar ME, Eschwège E: The effect of metformin on the metabolic abnormalities associated with upper-body fat distribution. BIGPRO Study Group. Diabetes Care 1996;19:920-926. 